\title{
Directives de l'ASSM «Attitude face à la fin de vie et à la mort»
}

\section{Daniel Scheidegger}

Président de I'ASSM

Correspondance: lic. iur. Michelle Salathé, MAE

Responsable du ressort Ethique

ASSM

m.salathe[at]samw.ch
En mai 2018, le Sénat de l'ASSM a approuvé les nouvelles directives "Attitude face à la fin de vie et à la mort». Celles-ci abordent de nombreux thèmes qui n'étaient pas encore traités dans la version antérieure de 2004 - actualisée en 2013 et retirée en mai -, dont le domaine d'application était limité aux patientes et patients en fin de vie. Les nouvelles directives contiennent des lignes directrices concernant l'attitude à adopter face au désir de mourir ainsi que des nouveaux modèles de décision tels que l'Advance Care Planning. Ces nouveautés ont été accueillies très positivement lors de la consultation. En revanche, le chapitre concernant l'assistance au suicide a déclenché un débat controversé, entre autres dans le Bulletin des médecins suisses. En octobre, l'ASSM a publié des messages clés concernant la réglementation de l'assistance au suicide, pour préciser certains éléments essentiels, parfois occultés ou relatés de manière approximative dans le débat public.

Le 25 octobre 2018, la Chambre médicale de la FMH a décidé de ne pas intégrer les nouvelles directives dans leur code déontologique et de maintenir la ver- sion de 2004. Le refus de la FMH est motivé par la décision de l'ASSM de prendre en considération le critère de la «souffrance subjectivement ressentie comme insupportable» comme condition possible pour une assistance médicale au suicide. La FMH considère ce critère comme une notion juridique indéterminée, qui apporte beaucoup d'incertitude pour le corps médical. L'ASSM a pris connaissance de cette décision et examinera minutieusement ses conséquences, en impliquant largement - comme pour l'adoption de ses directives - tous les organes compétents: la sous-commission responsable de l'élaboration des directives, la Commission Centrale d'Éthique, le Comité de direction et le Sénat. Il est important de poursuivre les réflexions sur ce thème, tant au sein des experts que de la société, et d'engager un débat public nuancé dans un climat constructif.

Les directives et les messages clés mentionnés sont publiés su le site web de I'ASSM: assm.ch/fin-de-vie-et-mort 